\title{
Acoso laboral e inequidad de género una problemática que afecta la comunicación de una organización
}

\section{The mobbing and gender inequality a problematic that affects communication in an organization}

\author{
Danna Valentina Castañeda - Hernández ${ }^{1}$, Elkin Andrés Guzmán - Roa ${ }^{2}$, Leidy Giovana Fernández - \\ Chavarro $^{3}$, Diego Alejandro Parada - Romero ${ }^{4}$, Harrinton Yesed Casanova - Rodríguez ${ }^{5}$ \\ ${ }^{1}$ Facultad de Negocios, Gestión y Sostenibilidad, Institución Universitaria Politécnico Grancolombiano, Colombia. \\ ORCID: https://orcid.org/0000-0001-8030-8942, E-mail: dacastaneda12@ poligran.edu.co \\ ${ }^{2}$ Facultad de Negocios, Gestión y Sostenibilidad, Institución Universitaria Politécnico Grancolombiano, Colombia, \\ ORCID: https://orcid.org/0000-0001-6093-7944, E-mail: elguzman2@poligran.edu.co \\ ${ }^{3}$ Facultad de Negocios, Gestión y Sostenibilidad, Institución Universitaria Politécnico Grancolombiano, Colombia, \\ ORCID: https://orcid.org/0000-0002-6882-1847, E-mail: lefernandez8@poligran.edu \\ ${ }^{4}$ Facultad de Negocios, Gestión y Sostenibilidad, Institución Universitaria Politécnico Grancolombiano, Colombia, \\ ORCID: https://orcid.org/0000-0001-5066-3943, E-mail: diparada4@poligran.edu.co \\ ${ }^{5}$ Facultad de Negocios, Gestión y Sostenibilidad, Institución Universitaria Politécnico Grancolombiano, Colombia, \\ ORCID: https://orcid.org/0000-0001-5164-6563, E-mail: hacasanova@poligran.edu.co \\ Cómo citar: Castañeda - Hernández, D. V., Guzmán - Roa, E. A., Fernández - Chavarro, L. G., Parada - Romero, D. \\ A., \& Casanova - Rodríguez, H. Y. (2020). Acoso laboral e inequidad de género una problemática que afecta la \\ comunicación de una organización. Revista Científica Profundidad Construyendo Futuro, 13(13), 49-61. \\ https://doi.org/10.22463/24221783.2613
}

Recibido: 09 de Mayo de 2020 / Aprobado: 15 de Junio de 2020

\begin{abstract}
Resumen
Varias organizaciones en Bogotá sufren de la inequidad laboral especialmente hacia la mujer, durante el desarrollo de este documento se empleó una encuesta a varias personas que trabajan en empresas "a b y c", con el objetivo de determinar si al haber un tipo de acoso laboral dentro de la organización esta hace que la comunicación se vuelva nula entre sus integrantes. en el estudio que se realizó se evidencio que las mujeres son más propensas a sufrir el acoso laboral debido a que se considera que son el género más débil haciendo que en muchos casos las empresas no tomen las decisiones adecuadas y por lo que se puede decir que al presentarse este tipo de problemas entre empleados y empleadores este puede perjudicar en el rendimiento de toda la organización.
\end{abstract}

Palabra clave: Acoso laboral, Comunicación, Daño psicológico, Mujer, Teoría del comportamiento.

\begin{abstract}
Several organizations in Bogotá suffer from labor inequality, especially towards women. During the development of this document, a survey was used of several people who work in companies "a, b and c" with the aim of determining whether, as there is a type of workplace harassment within of the organization this makes communication become null between its members and in turn causing psychological damage that can affect them in their day. In the study that was carried out, it was evidenced that women are more prone to bullying due to the fact that they are considered to be the weakest gender, causing in many cases companies not to make the right decisions and, therefore, it can be said that
\end{abstract}

*Autor para correspondencia

Correo electrónico: dacastaneda12@ poligran.edu.co

La revisión por pares es responsabilidad de la Universidad Francisco de Paula Santander Ocaña

Artículo bajo licencia CC BY-NC (https://creativecommons.org/licenses/by-nc/4.0/) 
When this type of problem occurs between employees and employers, it can affect the performance of the entire organization.

Keyword: Harassment at Work, Communication, Psychological Damage, Woman, Behavioral Theory.

\section{Introducción}

La presente investigación aborda el acoso laboral en contra de la mujer en las organizaciones que se ve frecuentemente, afectando el comportamiento y la comunicación de las personas que sufren este paradigma, se encuentra normalizado y regulado, y siendo un tema de cumplimiento obligatorio en lo que respecta a la salud ocupacional y en particular al riesgo psicosocial, aún se encuentra una gran resistencia en las organizaciones, lo que evidencia ausencia de información y probablemente temor para enfrentar realidades culturales en la empresa, que involucren la competencia del líder y sus efectos en el desarrollo del talento humano y finalmente la resistencia en las organizaciones para identificar comportamientos, saber cómo las empresas permiten este fenómeno que se da en el ámbito laboral tipo de discriminación, donde esta se ve afecta enormemente. Al comportamiento de las mujeres llegando así a un daño que puede llegar a largo plazo a ser psicológico y causar grandes afectaciones en este medio, es por eso que se profundiza la indagación desde la perspectiva de la sociología.

Rojas (2005) La discriminación laboral, hacia lo cual indica, las empresas tienen un gran defecto del acoso laboral donde los empleados se comportan de una manera distinta cuando hay un tipo de acoso laboral o el jefe hace explotación laboral, las personas no les gusta hacer lo que le ordene el empleador, lo hacen de mala gana o por necesidad, sumado a esto que en el país hay un gran porcentaje desempleo, y es muy difícil encontrar trabajo, más ahora que la tecnología está avanzando, remplazando al ser humano, y dejando atrás la manera en la que se ve el mundo laboral hoy en día.

Esto quiere decir que las empresas se aprovechan de lo que está pasando y solo quieren que los empleados trabajen gratis, humillados, tanto así que algunos de ellos llegan a punto de causar en ellos una depresión a nivel general y en su día a día, lo cual está afectando su salud mental por la falta de oportunidades, y esto sumado al acoso laboral vivido por algunas de estas personas con el impedimento de contar que está ocurriendo.

De acuerdo con Bocanegra (2015) los empleados son una parte esencial de la empresa, pues son ellos los que hacen crecer la compañía, promueven una adaptabilidad al mercado, tienen conocimiento más profundo del área de trabajo y como esta es manejada, es entonces que las empresas privadas deberían respetar más a sus empleados porque ellos son el musculo de una compañía, si los trabajadores se ven afectados la compañía también.

Por otro lado, afirma Peñaranda (2020) que en la naturaleza se presentan comportamientos, incluyendo los sistemas varía de una manera muy grande, así mismo, existe caos y orden conviviendo en diferentes proporciones. En este trabajo se presenta una revisión de los fundamentos relacionados al comportamiento de una persona con y una semejanza a la teoría trabajada, es decir se presenta una breve reseña acerca del 
surgimiento de la Teoría del comportamiento, y su conexión a las condiciones para que un sistema sea considerado caótico para las personas que llevan un trabajo donde no hay una estigmatización hacia las personas.

Lopez, Lopez y lopez (2016) en los objetivos de esta investigación, se encuentran diferentes pensamientos en los cuales se van a nombrar como el pensamiento que se tiene acerca del acoso laboral, y cómo influye en el comportamiento de las personas desde sus inicios, además de como las personas se adaptan a un cambio en las organizaciones dentro de una empresa rodeada por el acoso, y como es la vida de un individuo en el ámbito laboral, se hablará de como las empresas muchas veces no tienen en cuenta a sus empleados lo cual causa la afectación en la salud de los trabajadores y la baja productividad.

¿Por qué al haber acoso laboral dentro de las empresas hace que la comunicación se vuelva nula entre los mismos empleados interfiriendo en la producción de la empresa?

\section{Marco teórico}

\subsection{Antecedentes}

De acuerdo con la teoría del comportamiento la cual busca entender como las personas actúan dentro de su entorno laboral; para ello se enfoca en un estudio de investigación en el acoso laboral de acuerdo con el autor Bocanegra (2015) da un análisis conceptual de las actividades de los empleados en un ambiente laboral solo se expresa silencio a la hora de estar bajo presión sin entender el daño causado tanto a la persona como a la empresa, así afectando la comunicación y el comportamiento de las personas que trabajan bajo este entorno.
La teoría del comportamiento busca entender como los empleados se comportan dentro de una organización mediante las diferentes teorías planteadas por distintos autores que buscan dar respuesta a esta incógnita, en donde el comportamiento organizacional depende de distintos factores.

El ambiente laboral desde allí se puede decir de alguna manera que las personas en cuanto estén en mejor entorno más proactivas podrán ser dentro de la organización, a esto también se le sumaria, como es la vida laboral de cada individuo ya que cada uno tiene una función específica en donde se le pide una función distinta a los demás empleados.

Muchas de las empresas lo que buscan es poder reflexionar en cuanto al ámbito laboral que viven en sus organizaciones en el comportamiento de los empleados, buscan responder que podrían estar haciendo bien o mal, de acuerdo con los autores (Kameta, R.solaya, \& Prado, 2019)

Con todo este proceso se estudia una situación que es algo incómoda para los trabajadores pero que a la vez es necesaria, que es el objetivo de caracterizar el estatus de la variación en sistemas con dinámicas y variedades determinadas, la investigación del pensamiento y lenguaje.

Los autores Zabaleta, Micco, Gonzalez \& Et.al (2009) dentro del ámbito psicológico analiza el comportamiento de una persona estudiando la conducta de los empleados frente al problema de acoso laboral y como este afecta las emociones, de cada uno de ellos. 


\subsection{Teorías del comportamiento}

Los autores resumen Camacho \& M.Vazquez (2015) desde el punto de vista la teoría de caos se puede determinar con cierta población de personas, que podrían perjudicar el ambiente laboral que se enfoca en el daño de la empresa empezando con la convivencia de ellos representa, comportamientos de los cuerpos, describe e identifica el caos y el orden llevados a la teoría del comportamiento.

Lopez, Lopez y lopez (2016) se encuentra que varias mujeres en el mundo son afectadas psicológicamente según las estadísticas del acoso laboral, las mujeres tienen un $20 \%$ más alto que los hombres en recibir abuso emocional y psicológico y se evidencia que las mujeres se ven muy afectadas en el acoso laboral, determinando una falta de interés hacia el trabajo que está ejerciendo y esto puede afectar a la compañía directa o indirectamente como desde el ámbito laboral y psicológico puede afectar el comportamiento de ellas.

De este modo cuando en una empresa el ambiente organizacional es malo tiende a tener problemas de producción y suelen registrarse mayores conflictos interpersonales (Lynn, Rochelle, \& Weatherstondeborah, 2015) la salud mental infantil desde una temprana edad puede influir en comportamiento laboral que pueda llegar a tener una persona. En donde se busca entender como desde la crianza de los padres puede influir en la personalidad que llegue tener cada individuo en cualquier sitio.

Pero la crianza no es el único factor que puede influir en el comportamiento de una persona ya que este también depende del estrés laboral que este manejando, debido a que muchas personas que están dentro de la organización están manejando distintas emociones las cuales pueden llevar a un estrés severo y esto sucede debido a problemas personales o problemas dentro de la misa empresa. De acuerdo con (L.Cartillo, 2018)El problema por la salud mental se ha convertido en un factor importante llevando a cabo un análisis de las ciencias investigativas, llegando a debatir sobre el comportamiento del ser humano.

Las personas por lo general tienen cierto tipo de estrés debido a la carga laboral y necesitan al mismo tiempo de un buen líder, esto debido a que si se cuenta con un buen liderazgo podrá motivar a los empleados hacer más productivos en su campo y esto comparando los diferentes tipos de liderazgo se puede o no evidenciar el ausentismo del mismo (F.Leon, O.Morales, J.Ramos, \& et.al, 2017) Este tipo de comportamientos que se pueden presentar dentro de la organización pueden llegar a convertirse en una especie de acoso laboral en donde en muchos casos las víctimas son las mujeres.

$\mathrm{Al}$ ser las mujeres la principal víctima de acoso dentro de las organizaciones se busca analizar los programas internacionales de la red mujer y hábitat de América latina y extraer algunas líneas de acción para la incorporación de una perspectiva de derechos en la formulación de las políticas públicas todo esto dicho por Monrroy y Jaramillo (2017) el acoso hacia la mujer dentro del campo laboral es un tema de mucha controversia para todos, basándose en como desde el ámbito laboral y psicológico puede afectar el comportamiento de ellas y como a lo largo de la historia aún existen personas que creen que las mujeres no merecen un 
trabajo en algún tipo de empresa, sino que deben estar en casa sin ningún derecho a trabajar, estos son algunos de los maltratos que reciben las mujeres por parte de sus compañeros o empleadores dejando trastornos psicológicos en donde en muchas ocasiones las mujeres deben acudir a cualquier tipo de ayuda psicológica. (M.Trinidad, M.Ramirez, M.Anevar, \& Et.al, 2018)

\subsection{Tendencias}

La presente investigación es determinar las principales variables que se relacionan con el concepto de calidad de vida laboral, lo cual se realizó mediante una búsqueda de artículos científicos publicados en los últimos siete años, en función de los términos calidad de vida laboral, el comportamiento dentro de una empresa por parte de los empleados puede depender de distintos factores como los son el estrés debido a la carga laboral, asuntos personales entre otros los adultos depende de cómo se formaron en su infancia $\mathrm{y}$ adolescencia y pues en algunas ocasiones este comportamiento puede influir dentro de las organizaciones debido a que la forma de ser de una persona puede afectar el ámbito laboral y dañando el ambiente dentro del mismo, haciendo surgir un posible acoso laboral hacia las mujeres que en muchas ocasiones no son solucionados.

Pando, C.Aranda, J.Salazar, \& Et.al (2016) en los textos consultados e investigados evidenciamos que las mujeres se ven muy afectadas en el acoso laboral, determinando una falta de interés hacia el trabajo que está ejerciendo y esto puede afectar a la compañía directa o indirectamente ya que las personas que sufren acoso laboral van al trabajo por necesidad y soportar este tipo de humillaciones.
Además del acoso laboral las mujeres también se han visto discriminadas en diferentes aspectos como lo es en el salario que ganan, esto debido a que se puede entender como una forma de machismo dentro de la organización, según el autor (Rojas, 2005) La discriminación por ingreso resulta evidente especialmente en el sector privado, el cual necesita una sacudida en términos de responsabilidad social, de equidad en general y equidad de género en particular. Si el sector empresarial no siente que la inequidad es un drama del país, la inequidad de género no lo conmueve.

Pero que debe hacer una empresa cuando reciba una queja por acoso laboral según Peñaranda (2020) primer paso es entender qué es el acoso laborar y de ahí se toman las medidas correspondientes; llevar la queja al comité de convivencia laboral y general la información al departamento de recursos humanos, para después plantear posibles soluciones.

Se refiere a dos entidades o personas que mantiene una relación y buena comunicación para la adaptación y convivencia a la hora de hacer una labor o actividad. En la actualidad vemos que la comunicación es más fácil pero menos efectiva en ciertos casos, aunque no estén muy claras las ideas y comportamientos de todas las personas hay cosas que debemos compartir para agrupar ideas y solucionar todo obstáculo en el ámbito laboral. Pero el acoso laboral no solo se presenta en organizaciones o empresas privadas también se presenta en los sectores públicos como es el caso de la enfermería u hospitales, esto debido a que en muchos de los casos, las enfermeras son acosadas por el uniforme que ellas pueden llegar a tener, un estudio realizado por (M.Morelo, Fuentes, \& linares, 2016) afirman que, se identificó 
aproximadamente un $20 \%$ que el personal de enfermería presenta diferentes tipos de acoso laboral, y se identificó que los casos más frecuentes lo sufren las mujeres menores a 30 años, poca trayectoria profesional y los turnos que puedan llegar a tener, causando diferentes consecuencias, como los son daños psicológicos, sociales y productivos.

El acoso laboral pude tener diferentes tipos de definiciones y se presenta de distintas maneras de acuerdo con el autor (M.Pando, C.Aranda, J.Salazar, \& Et.al, 2016) la definición de acoso laboral se entiende como un fenómeno social que trata sobre el abuso emocional y conductas agresivas y constantes en un entorno o ambiente de trabajo.

El acoso laboral va más allá de un maltratado creado en los ámbitos laborales, además de generar malos resultados en la producción o en una actividad laboral, todo genera maltrato psicológico (agrede, causa daño emocional, corrompe la tranquilidad de la persona y no la deja progresar) o genera un daño y un problema en las organizaciones, además de violar los derechos y normas de una organización.

\section{Objetivos}

\subsection{Objetivo general}

Conocer sobre el acoso frecuente hacia la mujer en una organización y observar si este tiene como consecuencia que la comunicación se vuelva nula y que se tengan daños psicológicos entre cada uno de los integrantes de dichas empresas.

\section{Metodología}

\subsection{Método}

Por medio de la investigación a tres empresas del país "a, b y c” (No se dirá el nombre de estas empresas por seguridad de las mismas), se quiere da a conocer una visión acerca de cómo los comportamientos ocasionados por el acoso laboral en la empresa y su forma de comunicación pueden variar, pues se tendrá como eje principal el que cada persona tiene una forma diferente de expresar y sentir cada tipo de maltrato en las tres empresas seleccionadas, con la recolección de datos se podrá observar si realmente el comportamiento de todas las personas es diferente o si al contrario puede llegar a ser igual; con esto se quiere llegar a la conclusión de cómo a partir de esta investigación se puede hallar el mejor método o una mejor línea de ayuda para que estas empresas mejoren la comunicación, para que esta se vuelva efectiva y sea mucho más fácil el poder identificar los casos de acoso laboral ya que estos a lo largo de los años se han vuelto muy frecuentes en cada una de las empresas.

Dando un enfoque en las mujeres, pues estas son las que más sufren esta modalidad, es por eso que se llevará a cabo una investigación de enfoque cualitativo en donde se estudiará el comportamiento que tienen los hombres hacia las mujeres y las mismas entre si ya que de algún modo se puede llegar a presentar un tipo de cambio en el comportamiento de estas y su desarrollo a nivel íntegro y comunicativo en la empresa, identificando elementos y dinámicas de la comunicación relacionados con la interrupción, distorsión o ruptura de esta, también la descalificación, la denigración, insinuaciones hostiles y la ruptura de los 
Danna Valentina Castañeda Hernández, Elkin Andrés Guzmán Roa, Leidy Giovana Fernández Chavarro, Diego Alejandro Parada Romero, Harrinton Yesed Casanova Rodríguez

contactos sociales entre los casos de acoso laboral.

\subsection{Contexto y los participantes}

El contexto se dio en la universidad Politécnico Grancolombiano, se inició una orientación por medio del profesor de esta asignatura en el primer semestre del año 2020, nos dio instrucciones acerca de cómo hacer una investigación y cómo llevarla a cabo, de la cual surgió la idea de investigar acerca de la teoría del comportamiento dirigida al acoso laboral y esta se inicia pensando en la observación y exploración de tres empresas, clasificadas como " $a, b$, c" una vez definido que se llevará a cabo se procederá a interrogar a una serie de personas de dichas empresas ya nombradas en donde se les realiza una encuesta realizada por nosotros con preguntas rigurosas que interrogan acerca de cuánto se sabe del acoso laboral, si lo han sufrido, comportamiento de los demás compañeros, algún tipo de acoso laboral, etc. Además de la investigación en comprensión, evaluación , transformación y procesos socio psicológicos por dicho caso, en donde se desea conocer cómo es ambiente laboral de las empresas para tener una clara visión de cómo se podrían llegar a comportar las personas manejando el estrés laboral que se presenta debido a la gran carga laboral de las empresas escogidas, que muchas veces se le asigna a una sola persona y esto también se podría interpretar de algún modo como acoso laboral y del cual se quiere buscar la mejora en el ámbito laboral y organizacional con la finalidad de que dichas organizaciones crezcan y mantengan un nivel de producción exitosa, que contenga buenos entornos laborales y positivos ambientes para todos los empleados en general.

\subsection{Instrumento (s)}

Por medio de una encuesta se utilizará el siguiente link https://n9.cl/ys95 y un método con alcance descriptivo, se trabaja con tres empresas clasificadas por "a,b,c" esto con el fin de conocer el comportamiento de cada una de ellas en acoso laboral y la influencia que tiene para la comunicación efectiva, también investigando por medio del campo exploratorio para llegar a contrastar con la encuesta que se va a realizar, pues es importante conocer el comportamiento de cada uno de los empleados de dichas empresas, las cuales buscan dar respuesta a cada uno de nuestros interrogantes desde el punto de vista de personas con experiencia sobre el tema tratado es lo más importante, cabe resaltar que las preguntas serán enfocadas en el conocimiento de acoso laboral, las cuales pasarán por un proceso de verificación en el cual se hará una clasificación de los puntos más relevantes para encontrar respuesta a este conflicto, teniendo a su vez en cuenta la realidad de la sociedad y cuales dan una pauta de cómo esta puede hacer que los comportamientos en las personas se vean en su mayoría muy variados y diferentes, dependiendo variables como, el puesto de trabajo, estrato social, tipo de empresa, comunicación asertiva o nula, entre otros.

\subsection{Procedimiento}

Primera fase: Se realizó a través de plataformas digitales la elección de distintos artículos referentes al tema dado, estos se seleccionaron de manera tal que después se pudieran escoger los que más favorecían y aportaban para la investigación, pues de cada uno de ellos aportaría a dar mayor respuesta a los interrogantes acerca de esta problemática, no obstante, algunos de estos artículos por 
muy pequeños que fueran aportaron de manera precisa para hallar mejor la recolección de datos.

Segunda fase: Se interpreta cada una de las fases (artículos y encuesta) tomando en cuenta la perspectiva que las personas tienen en cuanto a este tema del acoso laboral hacia la mujer, relacionando así de la misma manera el cómo muchas veces las personas suelen o podrían quedarse calladas frente a estas injusticias, pues cabe resaltar que la encuesta fue realizada a hombres y mujeres para reconocer el cómo este es un tema de todos y no solo el de una sola población.

Tercera y última fase: Se inicia con la discusión acerca de los resultados obtenidos en la encuesta, se realiza un análisis por cada pregunta de la encuesta, para así tomar los puntos de vista de cada uno, la propuesta de una o más soluciones para esta problemática, sin descartar que estás soluciones se presentarán con un énfasis en la teoría tratada en esta investigación.

\section{Resultados}

Los resultados que se evidencian corresponden a la muestra seleccionada fundamentalmente aplicada a 30 personas que ocupan diversos cargos en organizaciones de Bogotá, a quienes por consideraciones de confidencialidad se dio tratamiento anónimo. La primera hablara acerca del género, para la investigación es muy importante a la hora de hacer una comparación con las respuestas de los siguientes enunciados, la cual contestaron 17 mujeres y 13 hombres en ese orden.

Para la siguiente pregunta, la cual a grandes rasgos mencionaba el si se ha sufrido de acoso laboral mostró que, en las mujeres el sí, fue por 4 de ellas, el tal vez 4 también y el no por 7. Para los hombres en cambio el sí, fue de 3 , el tal vez 0 , y el no 10 de ellos; esto demostrando en contraste que las mujeres son las que más sufren de acoso laboral en cada una de sus labores diarias, las cuales por medio de este acoso hacen más visibles las brechas laborales, pues impiden su desarrollo como personas libres ante todo en la sociedad, y que al contrarió deben vivir con un miedo cotidiano.

Para el siguiente enunciado el cual mencionaba si ha sido acosado/a sexualmente por el jefe muestra que, en las mujeres el sí, 2 de ellas lo han sufrido, el tal vez, 0 y el no 15 de ellas, en los hombres el sí, es 0, el tal vez, también 0 y el no, los 13 encuestados, nuevamente se puede observar como las mujeres son las más afectadas con este tipo de acoso que va de manera descendente desde la mayor autoridad hacía los puestos más bajos, causando así que el entorno de la empresa para las mujeres no sea el más próspero y bueno.

En la cuarta pregunta hace referencia si el acoso laboral a la mujer afecta a su comportamiento, esto demostró que en las mujeres sí lo sienten 14 de ellas, tal vez 2 y no solo una de ellas, para los hombres en cambio, sí 11 de ellos lo piensan, tal vez 2, y no ninguno de ellos, esta pregunta es muy importante, pues demuestra que los cambios en el entorno laboral y el comportamiento de estas personas que lo sufren pueden variar, según los resultados tanto hombres como mujeres piensan que así es presentando fuertes cambios en su comportamiento que a largo plazo pude causar un daño emocional y podría afectar al día a día de la persona.

En contraste con la anterior pregunta se presenta el mismo caso, con la diferencia de si afecta el comportamiento a nivel personal, 
este arrojo el sí en las mujeres predomina con 15 a favor, tal vez con 1 y el no 1 también, para los hombres de la misma manera el sí predomina con 10, el tal vez con 3 de ellos, y el no con 0 , en semejanza con el punto anterior se evidencia que el sufrir cambios de comportamiento si puede afectar la vida personal, en muchos casos la familia puede llegar a sentir grandes cambios que pueden llegar a causar una comunicación nula en ellos.

La pregunta seis menciona si la actitud del acosador puede dañar a la víctima psicológicamente, en las mujeres el sí es de 10 a favor, no sabe, 6 y el no únicamente 1 , para los hombres, los resultados muestran que el sí 6 de ellos lo creen, no sabe 6 igualmente y el no 1 de ellos, los encuestados demuestran que efectivamente el acoso va ligado al daño psicológico, el cual se ve involucrado por una afectación a nivel mental que sufre la persona, causando estrés, molestia, hasta la bipolaridad, que va ligado acudir a ayuda profesional, porque hay casos en los que él afectado no encuentra paz en ningún lado y no puede sentirse a gusto nunca.

La pregunta siete se profundizará más adelante, pues esta respuesta es de opinión personal y los resultados no son numéricos.

Siguiendo con el análisis; el enunciado de la pregunta ocho califican las acciones de acoso que cada persona puede hacer si esta se clasifican o no en una violencia extrema, 10 mujeres contestaron a favor, 5 contestaron tal vez, y 2 de ellas no, en los hombres 4 contestaron a favor, 7 de ellos tal vez y no 0 , aunque en este enunciado el sí predomina para ambos géneros, no se pude dejar atrás que el tal vez, va con casi la misma fuerza, esto ocurre porque el acoso laboral se puede presentar de muchas maneras y actitudes pero no se descarta que este acoso en su máximo esplendor causa una violencia extrema para los sujetos con esta afección.

Por otro lado, el noveno pregunta si la familia y amigos han estado apoyándolos si han sufrido este acoso, para las mujeres el sí es de 3, el tal vez 4 de ellas y el no 10 de las faltantes, en los hombres el sí en 2 de ellos, el tal vez únicamente 1 y el no 10 igual que las mujeres, muchas veces el acoso tiene causantes de tipo familiar que no obedece al trato cordial hacía la persona, en la sociedad muchas veces hay dos tipos de personas, las que hacen algo para que este algo suceda, y las que no hacen nada y aun así pasa, pero de lo que no se toma en cuenta es que solo se toma en la mayoría de los casos la opción uno y se prefiere estigmatizar lo que hizo la persona afectada para producir que le hicieran algo malo y es por eso aunque suene extraño la familia prefiera dar la espalda y no entrar en discusión con estos temas.

A continuación, el punto diez en su enunciado pregunta si usted denunciaría el acoso laboral, el sí de las mujeres es de 12, el tal vez 0 , y el no de 5 de ellas, por el lado los hombres el sí es de 11 a favor, el tal vez 0 de ellos al igual que en las mujeres y el no es de 2 de ellos, esta pregunta es muy importante porque demuestra que aunque la mayoría denunciaría el acoso, hay personas que no lo harían, lo cual da a entender que este tema no presenta la relevancia suficiente en la sociedad como para verla algo normal y que debe denunciarse, muchas veces y en los casos consultados, se observa cómo el miedo es fundamental para esto y como pude causar un gran trasfondo más de lo que se imagina, pues se tiene el interrogante de "si denuncio y pierdo todo" o "si mejor no denuncio y conservo mi empleo" es algo muy extenso, y a lo que se debe poner cuidado porque las personas muchas veces prefieren callar algo 
importante y tratar de seguir adelante para no perder nada como ellos creen. (Basado en tabla1).

Tabla 1. Encuesta

\begin{tabular}{|c|c|c|c|c|}
\hline Preguntas & Femenino & Masculino & $\begin{array}{l}\text { No } \\
\text { dice }\end{array}$ & $\begin{array}{l}\text { Total, } \\
\text { general }\end{array}$ \\
\hline \multirow[t]{2}{*}{ 1. Sexo } & 17 & 13 & 0 & 30 \\
\hline & No & Sí & $\begin{array}{l}\text { Tal } \\
\text { vez }\end{array}$ & $\begin{array}{l}\text { Total, } \\
\text { general }\end{array}$ \\
\hline \multirow[t]{2}{*}{$\begin{array}{l}\text { 2. ¿Ha sufrido de } \\
\text { acoso laboral? }\end{array}$} & 19 & 7 & 4 & 30 \\
\hline & No & $\mathrm{Si}$ & $\begin{array}{l}\text { Tal } \\
\text { vez }\end{array}$ & $\begin{array}{l}\text { Total, } \\
\text { general }\end{array}$ \\
\hline \multirow{2}{*}{$\begin{array}{l}\text { 3. ¿Ha sido } \\
\text { acosada } \\
\text { sexualmente por } \\
\text { parte de su jefe? }\end{array}$} & 28 & 2 & 0 & 30 \\
\hline & No & Sí & $\begin{array}{l}\text { Tal } \\
\text { vez }\end{array}$ & $\begin{array}{l}\text { Total, } \\
\text { general }\end{array}$ \\
\hline \multirow{2}{*}{$\begin{array}{l}\text { 4. ¿Cree que usted } \\
\text { que el acoso } \\
\text { laboral hacia la } \\
\text { mujer puede } \\
\text { afectar en su } \\
\text { comportamiento } \\
\text { laboral? }\end{array}$} & 1 & 25 & 4 & 30 \\
\hline & No & Sí & $\begin{array}{l}\text { Tal } \\
\text { vez }\end{array}$ & $\begin{array}{l}\text { Total, } \\
\text { general }\end{array}$ \\
\hline \multirow{2}{*}{$\begin{array}{l}\text { 5. ¿Cree que usted } \\
\text { que el acoso } \\
\text { laboral hacia la } \\
\text { mujer puede la } \\
\text { puede afectar en } \\
\text { su } \\
\text { comportamiento } \\
\text { personal? }\end{array}$} & 1 & 25 & 4 & 30 \\
\hline & No & No sabe & $\mathrm{Si}$ & $\begin{array}{l}\text { Total, } \\
\text { general }\end{array}$ \\
\hline \multirow{2}{*}{$\begin{array}{l}\text { 6. ¿La actitud de la } \\
\text { persona acosadora } \\
\text { existe intención } \\
\text { expresa de dañarla } \\
\text { psicológicamente? }\end{array}$} & 2 & 12 & 16 & 30 \\
\hline & No & Sí & $\begin{array}{l}\text { Tal } \\
\text { vez }\end{array}$ & $\begin{array}{l}\text { Total, } \\
\text { general }\end{array}$ \\
\hline \multirow{2}{*}{$\begin{array}{l}\text { 8. ¿Se pueden } \\
\text { calificar las } \\
\text { acciones de acoso } \\
\text { de "violencia } \\
\text { psicológica } \\
\text { extrema? }\end{array}$} & 4 & 14 & 12 & 30 \\
\hline & No & Sí & $\begin{array}{l}\text { Tal } \\
\text { vez }\end{array}$ & $\begin{array}{l}\text { Total } \\
\text { general }\end{array}$ \\
\hline $\begin{array}{l}\text { 9. Si usted fue o es } \\
\text { víctima de acoso } \\
\text { laboral, iA } \\
\text { recibida ayuda por } \\
\text { parte de familia } \\
\text { y/o amigos? }\end{array}$ & 20 & 5 & 5 & 30 \\
\hline
\end{tabular}

\begin{tabular}{|c|c|c|c|c|}
\hline & No & Sí & $\begin{array}{c}\text { Tal } \\
\text { vez }\end{array}$ & $\begin{array}{c}\text { Total } \\
\text { general }\end{array}$ \\
\hline $\begin{array}{l}\text { 10. ¿Usted } \\
\text { denunciaría } \\
\text { acoso laboral? }\end{array}$ & 9 & 21 & 0 & 30 \\
\hline
\end{tabular}

En el análisis anteriormente leído, se resaltó que el enunciado número siete nos presentaba una pregunta problema, en la cual cada una de las personas debía responder de manera individual el que harían en esa situación, demuestra que en su gran mayoría las personas tomarían acciones al respecto, como: denunciar al acosador, ayudar a la persona, impedir a que siga ocurriendo, entre otros, pero también es importante el resaltar que otras simplemente no harían nada porque no es problema de ellos y no deben tomar en cuenta un asunto que no les afecta (Basado enunciado 1).

7. ¿Qué puede hacer usted al respecto si ve acoso laboral hacia
una mujer dentro de su entorno?
Denunciar a la persona
Dirigirme a las autoridades competentes para solucionar la
situación
Llamar a la policía
Interferir
Denunciar, hablar con la persona que está siendo afectada
Denunciar
Impedir que pase Al alertar a todos que está pasando,
Nada, la persona que sufre de acoso es la que debe tomar la
decisión de hacer algo o no
Buscar alternativas para denunciar el acoso que la persona está
viviendo
Denunciar
Aconsejarla para que denuncie el acoso ante las entidades
correspondientes
Denunciarlo
Denunciarlo
Denunciar

\section{Enunciado 1.}

Para finalizar, se quiso de la misma manera que el punto siete observar el comportamiento de las personas con un caso hipotético que puede ocurrir en la vida real y como estas actuarían o se sentirían respecto a este, las personas tuvieron en general la misma respuesta, pues casi todas no están desacuerdo con la decisión de Martha y creen 
que debió tomar otras acciones, aunque no se debe olvidar que unas cuantas personas piensan que hizo lo correcto simplemente por evitar problemas.(Basado en enunciado 2).

\begin{tabular}{l} 
11. Cree usted que Martha hizo lo correcto? ¿Por qué? \\
No porque eso es violación de integridad de una persona \\
No, al ceder ante la intimidación solo le dio paso a que la conducta \\
del jefe se repita con una nueva compañera de trabajo, pues ahora \\
cree que puede hacerlo sin nunca recibir las consecuencias \\
Pienso que Martha debió tener una reacción inmediata ante la \\
situación y poner en su lugar al jefe. Obviamente no justifico las \\
acciones de este hombre ya que actuó sin ningún respeto y \\
profesionalismo hacia Martha \\
Tal vez necesita un ascenso \\
No, sin embargo, si jefe no va a cambiar de pensamiento y actitudes \\
por el hecho de hablar o denunciar. Lastimosamente este tipo de \\
abusos los viven muchas mujeres y diariamente. \\
Martha debió informar y denunciar Del acoso causado por su jefe \\
para que se tomaran las medidas necesarias y dejar como último \\
recurso la carta de renuncia. \\
Creo que está mal que Ella lo hubiera permitido Al principio, porque \\
así Ella lo haya hecho por miedo, Al hacer eso El entiende que Ella \\
accede. \\
Mal, porque dejo avanzar la situación de acoso, debió poner un alto \\
en el principio de los hechos \\
No, debió denunciar \\
¡Si y no! Si por sanidad mental no debería seguir en la misma \\
compañía y no porque no hizo nada para impedir que el señor \\
siguiera actuando de la misma forma con otras personas \\
No, ella debió denunciar lo sucedido. \\
Debió denunciarlo con él área de talento humano y meterle una \\
demanda \\
No hizo lo correcto, lo correcto era denunciar desde el principio \\
No, desde el primer momento debió hacer algo, al renunciar solo \\
demostró que era intimidada por él y dio la oportunidad de que el jefe \\
hiciera lo mismo con otras mujeres. \\
No actuó correctamente por qué permitió que el acoso contra ella se \\
presentará de manera prolongada. Si ella hubiera frenado las cosas \\
desde un principio probablemente no hubiera ocurrido mucho \\
No porque le está dando. La razón al jefe en vez de denunciar \\
\hline
\end{tabular}

\section{Discusiones}

En la actualidad las empresas se ven enfrentadas por el aumento en la presentación de quejas acerca del acoso laboral, que se ven comprendidas por las personas que son afectadas en su gravedad con casos donde la inestabilidad emocional se ve mayormente presente en estas (M.Peñaranda, 2020), en contraste con esta afirmación se puede obtener un análisis profundo el cual da como principal semejanza que los resultados en esta investigación acentúan que realmente todos estos casos de acoso dan como consecuencia un gran deterioro emocional y que causan que las personas que sufren este maltrato no vuelvan a ser las mismas, es decir, su salud mental se ve gravemente afectada, haciendo que su vida diaria sea menos proactiva y eficiente, además el ser humano promedio prefiere ser dirigido, desea evitar la responsabilidad, tiene relativamente poca ambición, desea seguridad por encima de todo (Castillero, 2020) dado que esta investigación muestra como el ser humano puede no tener la habilidad de contar las problemáticas de su día a día y mucho menos las que le ocurren en el entorno de trabajo este se puede convertir en un ser sin razón, haciendo que no se tenga en cuenta su opinión y que en casos aún más peores este se le considere como un ser que no puede expresar lo que le ocurre, así que se da una realidad en la que se prefiere no contar lo que pasa por conservar su empleo e ingresos para su familia.

Debido a esta característica humana de desagrado por el trabajo, la mayoría de la gente debe ser coaccionada, controlada, dirigida, amenazada con castigos para que haga el esfuerzo necesario para el logro de los resultados organizacionales (Castillero, 2020) en la actualidad se considera al ser humano con un ser robótico que solo sigue órdenes y las acepta sin tener otra opción u opinión, pero frente a esto se debe iniciar a tener una mejor conciencia de como el día laboral tendría que ser, proponiendo cambios en este ámbito para que todo mejore y las personas tengan una facultad de sus deseos y decisiones más contralada, para lograr así que las problemáticas en la empresa por no tener una buena comunicación varíen a tal modo de que esta se vuelva más relevante e importante, haciendo que la organización siga por su buen camino a nivel operativo, pero con un 
desarrollo encaminado a la igualdad de opiniones.

Por otro lado, las mujeres viven en permanente período de prueba en el trabajo (C.lopez, 2015) las mujeres se ven directamente conectadas con el acoso laboral que en muchas ocasiones se presenta diariamente, para colmo de la sociedad estas son las más afectadas, y las que tienen problemas que son mayormente ocultados porque no tienen la opción de expresar lo que sienten u ocurre, pues casi nunca se llega a una solución efectiva, que haga pensar que las cosas están cambiando y que se puede notar una variación en estos índices de acoso, pero lo que sí se puede es iniciar a presentar mejores acciones y a incrementar la importancia de la mujer en la sociedad, la cual tiene derecho a expresar lo que ocurre, sin dejar de lado que se obtenga un resultado efectivo frente a este.

\section{Conclusiones}

Al hablar acerca de la afectación del ambiente laboral en la empresa, el cual es ocasionado por el acoso laboral se puede concluir que, con los estudios realizados a las empresas, estas si son susceptibles a sufrir una inestabilidad laboral, pues este tipo de acoso afecta a todos, y no hace que la empresa rinda lo mismo que debería rendir.

Se puede ver como la brecha entre los hombres y las mujeres es muy grande, pues ellas son las más afectadas al respecto y son las que más involucradas están en el tema. Las empresas estudiadas muestran y presentan que los problemas psicológicos están presentes de una manera dominante cuando se sufre de acoso laboral, pues en algunos casos como se ha estudiado las personas que sufren esto, sufren de desórdenes en su personalidad, y cambios determinantes en su vida personal y laboral.

La desinformación en cuanto a este tema es muy grande, además suele pensarse que este ni existe, y al no verse influyente ante la sociedad no se toman realmente medidas para que esto cambie, causando la comunicación nula en las empresas que lo sufren, pues muchas veces se prefiere el no hablar de lo que ocurre.

\section{Referencias}

A.Lopez, G.Lopez, \& M.lopez. (2016). Estilos de liderazgo con tendencia al acoso laboral. Revista.utp, 7.

Bocanegra, J. (2015). Caos de ser silencio . Redalyc.org, 16.

C.lopez. (2015). genesis y problematizacion de la ley de acoso laboral en colombia . Bogota: Redalyc.

Castillero, O. (2020). psicologia y mente . Obtenido de psicologia y mente : https://psicologiaymente.com/organiz aciones/teoria-x-teoria-y-mcgregor

F.Leon, O.Morales, J.Ramos, \& et.al. (2017). Liderazgo orientado a la gente en callcenters . Scielo.org, 217.

Kameta, S., R.solaya, \& Prado, C. (2019). Analisis de teorias y la nueva era de las organizaciones adaptandose al ser humano. Vlex, 9-30.

L.Cartillo. (2018). Porque es importante fortalecer la autoestima en ambito laboral. Repositorio alejandria, 50. 
M.Monrroy, \& Jaramillo, F. (2017). violencia urbana la red mujer habitat america latina caso colombia . cidob, 23.

M.Morelo, Fuentes, M., \& linares, J. (2016). acoso laboral entre personal de enfermeria . Redalic.org, 26.

M.Pando, C.Aranda, J.Salazar, \& Et.al. (2016). PREVALENCIA DE VIOLENCIA PSICOLÓGICA Y ACOSO LABORAL EN TRABAJADORES DE IBEROAMÉRICA. Redalic.org, 8.

M.Peñaranda. (11 de febrero de 2020). legisGestionHumana.com. Obtenido de legisGestionHumana.com: http://www.gestionhumana.com.logi nbiblio.poligran.edu.co:2048/gh4/Ba ncoConocimiento/Q/queja_de_acoso/ queja_de_acoso.asp

M.Trinidad, M.Ramirez, M.Anevar, \& Et.al. (2018). Acoso laboral a las mujeres en las maquiladoras, ¿mito o realidad? Fuente Académica Premier, 32.

O.G.Zabaleta, L.D.Micco, C.M.Gonzalez, \& Et.al. (2009). Ruido 1 / f d omplementado en FPGA . 4.

R.Camacho, \& M.Vazquez. (2015). Geometría fractal, Teoría del caos, y sus aplicaciones en la Industria Petrolera. ingenieria petrolera , 718739.

Rojas, J. (2005). persiste la discriminacion laboral hacia la mujer . legal.legis, 5 . 